\title{
New Links between mRNA Polyadenylation and Diverse Nuclear Pathways
}

\author{
Dafne Campigli Di Giammartino, and James L. Manley*
}

The 3' ends of most eukaryotic messenger RNAs must undergo a maturation step that includes an endonuc-leolytic cleavage followed by addition of a polyadenylate tail. While this reaction is catalyzed by the action of only two enzymes it is supported by an unexpectedly large number of proteins. This complexity reflects the necessity of coordinating this process with other nuclear events, and growing evidence indicates that even more factors than previously thought are necessary to connect $3^{\prime}$ processing to additional cellular pathways. In this review we summarize the current understanding of the molecular machinery involved in this step of mRNA maturation, focusing on new core and auxiliary proteins that connect polyadenylation to splicing, DNA damage, transcription and cancer.

\section{INTRODUCTION}

In the past decades, biochemical studies of individual $3^{\prime}$ processing factors have contributed greatly to our understanding of the molecular mechanisms underlying the maturation of mRNA $3^{\prime}$ ends. $3^{\prime}$ end processing of almost all eukaryotic mRNAs, with the exception of metazoan histone mRNAs, consists of two tightly coupled steps: hydrolysis of a phosphodiester bond, referred to as cleavage, and addition of a polyadenylate tail to the $3^{\prime}$ hydroxyl of the upstream cleavage product. This relatively simple process is mediated by a very elaborate set of proteins, which bind to specific sequences on the pre-mRNA and, once assembled correctly, direct cleavage at the correct location (reviewed in Proudfoot, 2011; Xiang et al., 2014). The core machinery includes four multi-subunit protein complexes (Fig. 1) (reviewed in Mandel et al., 2008): cleavage and polyadenylation specificity factor (CPSF), cleavage stimulation factor (CstF), cleavage factors I and II (CFI and CFII) as well as additional proteins such as PAP (poly(A)polymerase), symplekin, the C-terminal domain of the RNA polymerase II largest subunit (CTD) and other newly char-

Columbia University, Department of Biological Sciences, New York NY, 10027, USA

*Correspondence: jlm2@columbia.edu

Received 25 June, 2014; accepted 28 June, 2014; published online 1 August, 2014

Keywords: 3' end processing, cleavage, polyadenylation, pre-mRNA acterized factors discussed below. The most conserved ciselement, the AAUAAA sequence, is located upstream of the cleavage site, and is recognized by CPSF; variants of this motif are frequently associated with alternative usage of poly $(A)$ sites. Downstream of the cleavage site is a G/U-rich element, which is recognized by CstF. A third element, with the consensus UGUA, is often present in one or more copies upstream of the AAUAAA, and is recognized by CFI (Hu et al., 2005).

More than $70 \%$ of human genes encode primary transcripts that contain multiple polyadenylation sites (PASs) (reviewed in Di Giammartino et al., 2011; Elkon et al., 2013; Tian and Manley, 2013). These can be found either in internal introns, and therefore alternative polyadenylation (APA) will be coupled to alternative splicing producing different protein isoforms, or, more frequently, exclusively in the $3^{\prime}$ untranslated region (UTR), resulting in transcripts encoding the same proteins but with $3^{\prime}$ UTRs of different length, which can in turn affect the stability, localization, transport and translational properties of the mRNA. It is interesting that differential processing at multiple PASs can occur globally, and can be influenced by conditions such as differentiation and development, or by pathological conditions such as cancer (Ji and Tian, 2009; Ji et al., 2009; Lackford et al., 2014; Mayr and Bartel, 2009; Sandberg et al., 2008). Determining how changes in APA contribute to different physiological states is a major question for future research.

The complexity of the $3^{\prime}$ processing machinery has already suggested a number of links to other cellular processes. In this review we will discuss how several newly characterized proteins associated with the $3^{\prime}$ processing complex provide additional connections with various nuclear pathways and pathological conditions, such as splicing, DNA damage, transcription and cancer.

\section{NEW ADDITIONS TO THE HUMAN CORE 3' PROCESSING COMPLEX}

A few years ago, novel insights into the make-up of the polyadenylation complex were obtained from a purification and proteomic analysis of the entire $3^{\prime}$ processing complex in its functional form (Shi et al., 2009). The complex was purified at the "post-assembly" stage, after the complex had assembled on a substrate RNA but before any significant processing had occurred. Glycerol gradient sedimentation combined with RNA tagbased affinity purification led to identification of $\sim 85$ proteins that associated with substrates with intact processing signals, but not with RNAs containing AAUAAA mutations. These in cluded nearly all previously identified 3 ' processing factors, with the exception 


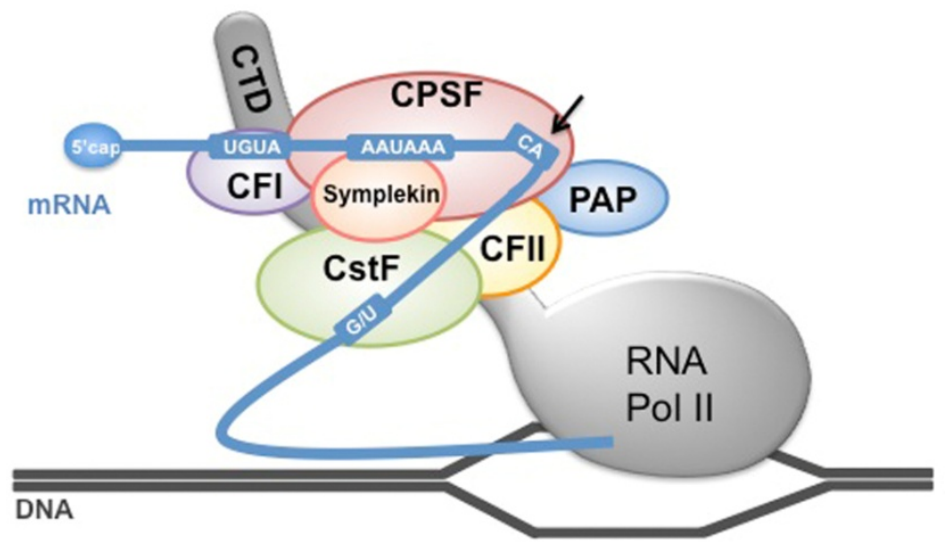

Fig. 1. The pre-mRNA $3^{\prime}$ processing complex. The core $3^{\prime}$ processing complex is composed of four multisubunit subcomplexes: CPSF, CstF, CFI and CFII together with additional factors such as symplekin, the CTD of RNA pol II and PAP. This machinery recognizes cis-elements within the pre-mRNA: CPSF binds to the AAUAAA sequence located upstream of the cleavage site (marked here with a black arrow), CstF recognizes G/U-rich elements downstream of the cleavage site and CFI binds the auxiliary sequence UGUA located upstream of AAUAAA. of Clp1, a component of CFII. In fact, the other identified CFII subunit, Pcf11, was barely detectable, indicating that CFII may associate with the complex only transiently. CFII is the least understood of the 3' cleavage factors: Pcf11 interacts with the RNA polymerase II CTD and is involved in transcription termination (Meinhart and Cramer, 2004), while Clp1 has been reported to have an RNA 5 '-kinase activity that is important for tRNA splicing and activation of siRNAs (Weitzer and Martinez, 2007). Therefore, even if not tightly associated with the $3^{\prime}$ processing complex, the function of CFII might be critical in connecting 3 ' processing to other nuclear pathways.

The canonical PAP (PAP $\alpha$ ) was also absent from the proteins purified with the $3^{\prime}$ complex. This suggests that it might be recruited at a later stage, which is in fact consistent with the earliest biochemical fractionation studies (Takagaki et al., 1988), or that another related protein might have taken its place. In this regard, it is interesting that $\mathrm{PAP} \gamma$ (also known as neoPAP; Topalian et al., 2001) was found to associate with the complex, although at low levels, indicating that PAP $\alpha$ and PAP $\gamma$ may play redundant roles. A recent crystal structure of PAP $\gamma$ shows that it shares a conserved catalytic binding pocket while residues at the surface are more divergent (Yang et al., 2014). The diversity in the C-terminal domain of these two proteins could contribute to differential regulation, as this region is known to be critical for regulation of PAP $\alpha$ activity through post-translational modifications (e.g., Colgan et al., 1996; Vethantham et al., 2008), and distinct isoforms can be produced that result from alternative splicing that affects this region (Zhao and Manley, 1996). Interestingly, PAP $\alpha$ has been reported to be phosphorylated throughout the cell cycle and downregulated by hyper-phosphorylation during M phase (Colgan et al., 1996) while PAP $\gamma$ did not show evidence of phosphorylation or alternative isoforms (Topalian et al. 2001).

An additional protein that was identified in the proteomic analysis mentioned above is CstF64 tau, a conserved paralog of CstF64. CstF64 tau was shown initially to be expressed specifically in the testis and brain (Wallace et al., 1999) and has been reported to mediate tissue-specific APA regulation (Li et al., 2012). However, its presence in the $3^{\prime}$ complex purified from HeLa cells hinted to a more general role in polyadenylation. Indeed, a recent study showed that CstF64 tau is widely expressed in mammalian tissues and has a similar RNA-binding pattern as CstF64 in vitro and in vivo (Yao et al., 2013). Also, the two proteins play redundant roles in alternative polyadenylation (APA) regulation such that depletion of either induces up-regulation of the other resulting in few changes in APA, but codepletion leads to greater APA changes (Yao et al., 2012). Nonethe- less, a significant difference between CstF64 and CstF64 tau is that the former binds symplekin with much higher affinity than the latter (Yao et al., 2013). Both proteins contain a "hinge" domain, initially shown to mediate CstF64 binding to symplekin (Takagaki and Manley, 2000), and both paralogs bind symplekin in vitro. However, the interaction with CstF64 tau is inhibited by its C-terminal Pro-Gly rich domain, which is the most divergent region between the two proteins. It is therefore possible that their association with the $3^{\prime}$ processing complex might be modulated by differential protein-protein interactions that depend on the Pro-Gly rich region, and these interactions, in turn, might reflect distinct functions in some aspects of mRNA 3' processing

The proteomic purification of the $3^{\prime}$ complex led to the identification of three proteins that were not previously implicated in mRNA $3^{\prime}$ processing in mammals but are related to known yeast 3' processing factors: PP1, WDR33 and RBBP6 (Fig. 2); each of these proteins is discussed below.

\section{PP1}

PP1 is a serine/threonine phosphatase homologous to Glc7, which in yeast is known to play a role in poly $(A)$ synthesis but not cleavage (He and Moore, 2005). Depletion of Glc7 in yeast has been shown to cause shortened poly $(A)$ tails in vivo; similarly, Shi et al. (2009) showed that HeLa nuclear extract (NE) depleted of PP1 displayed inhibited poly(A) synthesis activity, which could be restored by adding back recombinant PP1. Glc7 dephosphorylates Pta1 (He and Moore, 2005) and therefore PP1 is likely to dephosphorylate symplekin, the mammalian homolog of Pta1. Since symplekin acts as a scaffolding protein in the $3^{\prime}$ complex, it is possible that different states of phospho rylation of symplekin might affect its ability to interact with CPSF/CstF, ultimately modulating the efficiency of $3^{\prime}$ end formation. However, the phosphorylation status of symplekin is currently unknown.

Given the presence of PP1, it is not surprising that the PP1 regulatory protein, PNUTS, was found in the $3^{\prime}$ complex as well. PNUTS is known to form a stable complex with PP1 in mammalian cell extracts and has been shown to inhibit its catalytic activity (Kim et al., 2003). In addition, PNUTS has been shown to bind RNA in vitro (Kim et al., 2003), raising the possibility that PNUTS could have a direct function in recruiting PP1 to the $3^{\prime}$ complex.

PP1 is a multifunctional protein that plays a role in regulating different aspects of mRNA maturation. For example, PP1 is known to be required for the second step of pre-mRNA splicing, targeting specific snRNP proteins (Shi et al., 2006), while in yeast, 


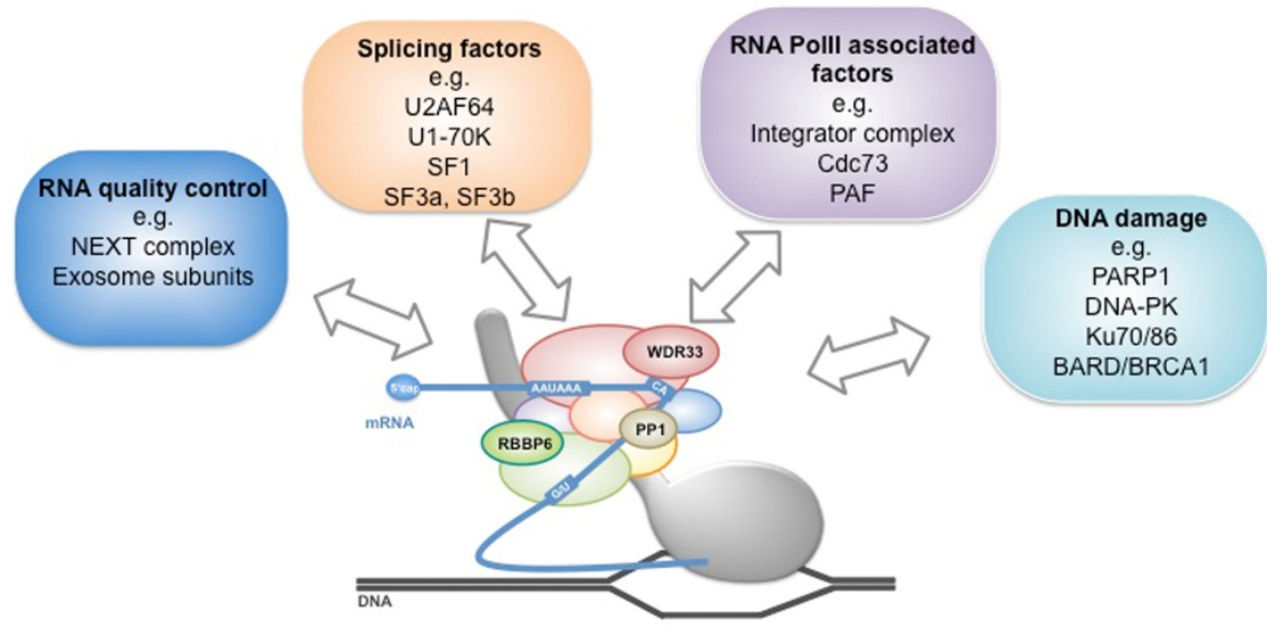

Fig. 2. Connecting $3^{\prime}$ process-ing with multiple nuclear pathways. WDR33, PP1 and RBBP6 are recently discovered $3^{\prime}$ processing factors that function in regulation of cleavage and polyadenylation. Additional proteins, as depicted in the figure, interact with the $3^{\prime}$ complex and function in connecting premRNA $3^{\prime}$ processing with other nuclear pathways such as RNA quality control, splicing, transcription and DNA damage.
Glc7 functions in mRNA export, through dephosphorylation of Npl3 (Gilbert and Guthrie, 2004). Future studies will reveal if PP1 can function to bridge $3^{\prime}$ end formation with such activities as splicing and mRNA export in mammalian cells.

\section{WDR33}

Another core subunit not identified in the mammalian $3^{\prime}$ processing complex until the above mentioned proteomics analysis is WDR33. Its size is similar to CPSF160 and this might explain why this protein previously escaped detection in other proteomic analyses that used only SDS-PAGE as a mean to separate proteins. The main characteristic of WDR33 are the seven WD40 repeats in its $\mathrm{N}$-terminus, a domain that is present in proteins involved in a wide range of cellular processes, as well as in the $3^{\prime}$ factor CstF50. The underlying common function of most WD40-repeat proteins is that they coordinate multi-protein complex assemblies, where the repeating units serve as a scaffold for protein interactions (Xu and Min, 2011). In addition, WD40 domains have also been reported to bind both ubiquitin (Pashkova et al., 2010) and phosphorylated Ser/Thr residues (Reinhardt and Yaffe, 2013). The yeast homolog of WDR33 is Pfs2, which has been shown to be essential for $3^{\prime}$ processing and might play a role in tethering the yeast CPF and CFIA complexes together (Ohnacker et al., 2000). Similarly to Pfs2, WDR33 was shown to interact with CPSF components and its depletion from HeLa NE abolished both cleavage and polyadenylation (Shi et al., 2009). It remains to be determined if WDR33 coordinates the interaction between CstF and CPSF in mammalian cells the same way as Pfs 2 does in yeast. For the future it will also be of interest to investigate whether the WD40 domain has a role in mediating the interaction of WDR33 with the other 3 ' factors and if this implicates Ser/Thr phosphorylated residues in the binding partners.

\section{RBBP6}

The third protein identified in the purification of the mammalian $3^{\prime}$ complex that shares homology with a known yeast $3^{\prime}$ processing factor is RBBP6. Its yeast counterpart is Mpe1, which is an essential gene. Mpe1 is an integral subunit of CPF (cleavage and polyadenylation factor) and is required for both cleavage and polyadenylation (Vo et al., 2001). RBBP6 was first identified in
1995 as a protein that interacts with the tumor suppressor $\mathrm{Rb}$ (Sakai et al., 1995) and later was shown to bind another tumor suppressor, p53 (Simons et al., 1997). The $\sim 250$ KD RBBP6 shares with Mpe1 three conserved domains in its N-terminus but has a unique long $\mathrm{C}$-terminal extension that mediates the binding to $\mathrm{p} 53$ and $\mathrm{Rb}$, raising the possibility that it may have a potential role in integrating $3^{\prime}$ processing with these nuclear pathways. RBBP 6 is able to regulate $3^{\prime}$ processing and it associates with the $3^{\prime}$ processing complex through a ubiquitin-like domain called DWNN (domain with no name). This domain is required for $3^{\prime}$ processing activity and is also expressed as a single domain protein (isoform3). Isoform3, which is down regulated in several cancers (Mbita et al., 2012), inhibits pre-mRNA 3' end cleavage by competing with the full-length RBBP6 for binding the core machinery (Fig. 3A) (Di Giammartion, D.C., Ogami, K., Li, W., Yashinskie, J.J., Hoque, M., Tian, B., and Manley, J.L., unpublished data). In the future it will be particularly interesting to better understand if isoform 3 can indeed function as a ubiquitinlike modifier and if this new type of modification is involved in regulating $3^{\prime}$ processing, for example by covalently attaching to core $3^{\prime}$ processing factors, thereby affecting their function. In addition, genome-wide analyses revealed that, following RBBP6 knockdown, there is a general lengthening in $3^{\prime}$ UTRs and a downregulation in mRNAs levels, in particular of transcripts with AU-rich $3^{\prime} U T R s$. More work is required to understand the molecular mechanism that underlies this specificity.

\section{OTHER NUCLEAR PROTEINS ASSOCIATED WITH THE 3' COMPLEX}

In addition to the known core $3^{\prime}$ processing factors and the other proteins discussed above, the proteomic analysis of the $3^{\prime}$ complex allowed detection of about fifty proteins that co-purified with the active complex (Shi et al., 2009) (Fig. 2). These include splicing factors such as U2AF65 and U1-70K, which were already found to mediate crosstalk between splicing and $3^{\prime}$ processing (Awasthi and Alwine, 2003; Gunderson et al., 1998; Vagner et al., 2000) and additional splicing factors that have not been shown yet to take part in $3^{\prime}$ processing. Among them are several proteins that bind the pre-mRNA at the $3^{\prime}$ splice site and participate in the assembly of early spliceosomal complexes, for example SF1, which binds to the branch point sequence (Berglund et al., 1997) and several subunits of the multiprotein complexes SF3a 


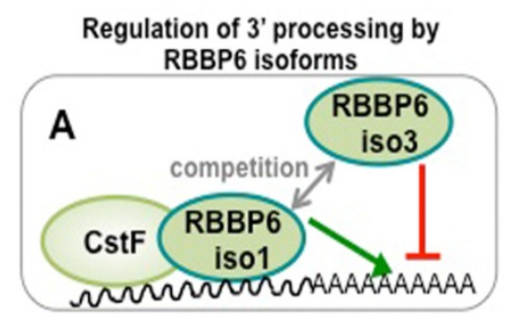

PARP1 inhibits polyadenylation during heat shock

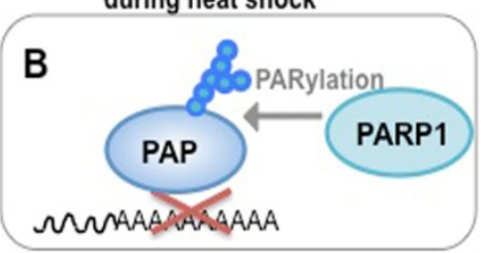

PAF mediates transcription-coupled polyadenylation

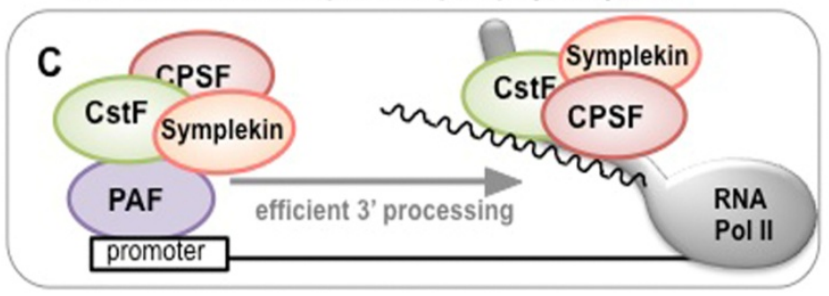

Fig. 3. Examples of $3^{\prime}$ processing regulation. (A) Isoform1 (iso1) of RBBP6 is required for efficient $3^{\prime}$ cleavage and associates with CstF through its DWNN domain. Isoform3 (iso3) of RBBP6, which is composed of the DWNN motif only and is downregulated in several human cancers, competes with iso1 for binding to CstF and inhibits $3^{\prime}$ processing. (B) PAF recruitment to promoter regions facilitates coordination of poly (A) factors with the transcription elongation complex, which results in enhanced efficiency of $3^{\prime}$ end formation. (C) During heat shock PARP1 PARylates PAP and causes its dissociation from $3^{\prime}$ ends of non-heat shock protein transcripts, resulting in polyadenylation inhibition. and SF3b, which are part of the U2 snRNP that bind in close proximity of the branch point (Gozani et al., 1996). Finding these factors in the active $3^{\prime}$ processing complex confirms the physical interaction between the polyadenylation and splicing machineries and may indicate that these proteins play a role in coupling splicing, for example of the terminal intron, to polyadenylation.

The tumor suppressor Cdc73 is a component of the RNA pol II-associated PAF complex and was found to associate with the $3^{\prime}$ processing complex as well. Around the same time a biochemical study was published which indeed confirmed that Cdc73 functionally associates with CPSF and CstF (Rozenblatt-Rosen et al., 2009). It was suggested that Cdc73 might regulate mRNA processing by facilitating the recruitment of $3^{\prime}$ factors to transcribed loci. Other evidence of PAF involvement in $3^{\prime}$ processing comes from yeast, where PAF was shown to affect poly (A) tail length (Mueller et al., 2004) and poly (A) site selection (Penheiter et al., 2005). In addition, another study (Nagaike et al., 2011) found a role of PAF in mediating stimulation of $3^{\prime}$ processing by transcriptional activators, confirming its potential role in bridging polyadenylation to transcription (Fig. 3B). It is, however, significant that none of the other five subunits of the PAF complex, only Cdc73, co-purified with the $3^{\prime}$ complex in the proteomic study (Shi et al., 2009), suggesting that Cdc73 might play a PAFindependent role in $3^{\prime}$ processing.

Another RNA pol II associated complex that was found to associate with the $3^{\prime}$ processing machinery is the Integrator. The Integrator mediates $3^{\prime}$ processing of $\mathrm{U} 1$ and $\mathrm{U} 2$ small nuclear RNAs (snRNAs) (Baillat et al., 2005), and several reports have emerged that suggest this complex might be multifunctional and play roles in various types of gene expression regulation beyond snRNA (Kapp et al., 2013; Takata et al., 2012; Zhang et al., 2013). Since almost all components of this complex were found in the proteomic analysis, it is very likely that the Integrator might have a yet undiscovered function in MRNA $3^{\prime}$ end formation as well.

Another interesting connection that stems from the proteomic analysis involves the NEXT complex (Lubas et al., 2011). NEXT is a multisubunit complex that is required for exosome-mediated degradation of noncoding RNAs such as promoter upstream transcripts (PROMPTs, also known as upstream antisense RNAs, uaRNAs), which are processed by the canonical $3^{\prime}$ cleavage machinery (Almada et al., 2013; Ntini et al., 2013) but targeted for rapid degradation by an unknown mechanism
(Richard and Manley, 2013). It is surprising that the three NEXT subunits, MTR4, ZCCHC8 and RBM7, were all found to associate with the $3^{\prime}$ complex (Shi et al., 2009). This could mean that NEXT might be involved in degradation of certain newly synthesized mRNAs, or other Pol II transcripts such as PROMPTs, by recruiting the exosome via interaction with the $3^{\prime}$ processing machinery. This might be similar to what occurs with the yeast TRAMP complex, which was shown to function in mRNA quality control by interacting with components of the exosome and stimulating degradation of aberrant mRNAs (Stuparevic et al., 2013). In fact some of the canonical exosome components were found as well in the proteomic analysis of the $3^{\prime}$ processing complex, including the catalytic subunit exosome10 (also known as Rrp6) and a number of non-catalytic subunits. The significance of these interactions will be an important topic for future study.

Finally, links between DNA damage response factors and the $3^{\prime}$ processing complex are especially intriguing. Previous studies have described similar connections, showing that $3^{\prime}$ processing is inhibited following DNA damage, concomitantly with an increased interaction between CstF50 and the BARD/BRCA1 complex (Kleiman and Manley, 1999, 2001). This interaction was later shown also to stimulate the deadenylation activity of PARN (poly(A)-specific ribonuclease) during DNA damage, leading to RNA degradation (Cevher et al., 2010). In addition, p53 also interacts with CstF50 and BARD1 and has an inhibitory effect on $3^{\prime}$ processing of housekeeping genes following UV treatment (Nazeer et al., 2011).

The proteomic study mentioned above identified DNA-PK as associated with the pre-mRNA $3^{\prime}$ processing complex. DNA-PK is a nuclear serine/threonine kinase that is comprised of a regulatory subunit, containing the Ku70/86 components, and a catalytic subunit, DNA-PK $\mathrm{K}_{\mathrm{cs}}$; interestingly, all of these subunits were found to associate with the $3^{\prime}$ complex. DNA-PK is a molecular sensor for DNA damage: it is involved in DNA nonhomologous end joining and is required for double-strand break (DSB) repair and VDJ recombination (reviewed in Collis et al., 2005). DNAPK must be bound to DNA to express its catalytic properties, but the fact that it is associated with the $3^{\prime}$ complex raises the possibility that RNA might activate it as well. It will be important to understand if DNA-PK functions to somehow connect the cellular response to DSBs to $3^{\prime}$ processing or if it has a separate function in the maturation of mRNA $3^{\prime}$ ends. One way to test this 
could be by using one of the several small molecule inhibitors of DNA-PK (Davidson et al., 2013), and determine whether it affects $3^{\prime}$ cleavage activity, either in the presence or absence of DNA damage. It is likely that one of the $3^{\prime}$ processing factors might be a target for phosphorylation by DNA-PK as several 3' factors are known to be phosphorylated (reviewed in Ryan and Bauer, 2008) but in most cases the kinase is unknown. Interestingly DNA-PK was shown also to phosphorylate and modulate PARP1 activity (Ariumi et al., 1999), another protein related to DNA damage that was identified in association with the $3^{\prime}$ complex.

PARP1 is an enzyme that catalyzes the post-translational modification known as Poly(ADP-ribosyl)ation (PARylation). PARP1 is known to take part in several cellular processes, including DNA damage detection and repair, chromatin modification and transcription (reviewed in $\mathrm{Ji}$ and Tulin, 2010; Krishnakumar and Kraus, 2010). Establishing the significance of the association of PARP1 with the $3^{\prime}$ processing complex, PARP1 was recently shown to PARylate PAP and to inhibit its activity in vitro (Di Giammartino et al., 2013). In vivo the modification can be detected after heat shock treatment, but not after activating PARP1 with IR radiation or $\mathrm{H}_{2} \mathrm{O}_{2}$. PARylation of PAP leads to polyadenylation inhibition due to the decreased ability of modified PAP to bind RNA (Fig. $3 \mathrm{C}$ ). Interestingly, a recent proteome-wide study showed that activation of PARP1 by $\mathrm{H}_{2} \mathrm{O}_{2}$ and MMS (but not by UV or IR radiation) leads to PARylation of several other $3^{\prime}$ processing factors such as PABPN1 and all CPSF subunits (Jungmichel et al., 2013), pointing to the possibility that PARP1 might be a general regulator of $3^{\prime}$ processing under a variety of conditions. For the future it would be very interesting to understand what determines the specificity of this regulation; for example, activation of PARP1 by different genotoxic stimuli might cause it to interact with different protein partners, which might then direct its enzymatic activity toward different targets.

In summary, it is becoming evident that the $3^{\prime}$ processing machinery is even more complex than previously thought. The need to connect polyadenylation with many cellular pathways such as transcription, splicing, cell cycle control and DNA damage, might explain why such an immense machinery is involved in regulating this relatively simple reaction. Coordination between the different steps of gene expression is critical for proper cellular function, and future studies need to focus on understanding in more detail the roles of the proteins discussed above.

\section{ACKNOWLEDGMENTS}

We would like to thank members of the Manley's lab for helpful discussion. Work from our lab was supported by R01 GM28983.

\section{REFERENCES}

Almada, A.E., Wu, X., Kriz, A.J., Burge, C.B., and Sharp, P.A. (2013) Promoter directionality is controlled by U1 snRNP and polyadenylation signals. Nature 499, 360-363.

Ariumi, Y., Masutani, M., Copeland, T.D., Mimori, T., Sugimura, T., Shimotohno, K., Ueda, K., Hatanaka, M., and Noda, M. (1999) Suppression of the poly(ADP-ribose) polymerase activity by DNA-dependent protein kinase in vitro. Oncogene 18, 4616-4625.

Awasthi, S., and Alwine, J.C. (2003). Association of polyadenylation cleavage factor I with U1 snRNP. RNA 9, 1400-1409.

Baillat, D., Hakimi, M.A., Naar, A.M., Shilatifard, A., Cooch, N., and Shiekhattar, R. (2005). Integrator, a multiprotein mediator of small nuclear RNA processing, associates with the C-terminal repeat of RNA polymerase II. Cell 123, 265-276.

Berglund, J.A., Chua, K., Abovich, N., Reed, R., and Rosbash, M. (1997). The splicing factor BBP interacts specifically with the premRNA branchpoint sequence UACUAAC. Cell 89, 781-787.

Cevher, M.A., Zhang, X., Fernandez, S., Kim, S., Baquero, J., Nilsson, P., Lee, S., Virtanen, A., and Kleiman, F.E. (2010). Nuclear deadenylation/polyadenylation factors regulate $3^{\prime}$ processing in response to DNA damage. EMBO J. 29, 1674-1687.

Colgan, D.F., Murthy, K.G., Prives, C., and Manley, J.L. (1996). Cellcycle related regulation of poly $(\mathrm{A})$ polymerase by phosphorylation. Nature 384, 282-285

Collis, S.J., DeWeese, T.L., Jeggo, P.A., and Parker, A.R. (2005). The life and death of DNA-PK. Oncogene 24, 949-961.

Davidson, D., Amrein, L., Panasci, L., and Aloyz, R. (2013). Small molecules, inhibitors of DNA-PK, targeting DNA repair, and beyond. Front. Pharmacol. 4, 5.

Di Giammartino, D.C., Nishida, K., and Manley, J.L. (2011). Mechanisms and consequences of alternative polyadenylation. Mol. Cell 43, 853-866.

Di Giammartino, D.C., Shi, Y., and Manley, J.L. (2013). PARP1 represses PAP and inhibits polyadenylation during heat shock. Mol. Cell 49, 7-17.

Elkon, R., Ugalde, A.P., and Agami, R. (2013). Alternative cleavage and polyadenylation: extent, regulation and function. Nat. Rev. Genet. 14, 496-506.

Gilbert, W., and Guthrie, C. (2004). The Glc7p nuclear phosphatase promotes mRNA export by facilitating association of Mex67p with mRNA. Mol. Cell 13, 201-212.

Gozani, O., Feld, R., and Reed, R. (1996). Evidence that sequenceindependent binding of highly conserved U2 snRNP proteins upstream of the branch site is required for assembly of spliceosomal complex A. Genes Dev. 10, 233-243.

Gunderson, S.I., Polycarpou-Schwarz, M., and Mattaj, I.W. (1998). U1 snRNP inhibits pre-mRNA polyadenylation through a direct interaction between U1 70K and poly(A) polymerase. Mol. Cell 1, 255-264.

$\mathrm{He}, \mathrm{X}$., and Moore, C. (2005). Regulation of yeast mRNA 3' end processing by phosphorylation. Mol. Cell 19, 619-629.

Hu, J., Lutz, C.S., Wilusz, J., and Tian, B. (2005). Bioinformatic identification of candidate cis-regulatory elements involved in human mRNA polyadenylation. RNA 11, 1485-1493.

Ji, Z., and Tian, B. (2009). Reprogramming of $3^{\prime}$ untranslated regions of mRNAs by alternative polyadenylation in generation of pluripotent stem cells from different cell types. PLoS One 4, e8419.

Ji, Y., and Tulin, A.V. (2010). The roles of PARP1 in gene control and cell differentiation. Curr. Opin. Genet. Dev. 20, 512-518.

Ji, Z., Lee, J.Y., Pan, Z., Jiang, B., and Tian, B. (2009). Progressive lengthening of $3^{\prime}$ untranslated regions of mRNAs by alternative polyadenylation during mouse embryonic development. Proc. Natl. Acad. Sci. USA 106, 7028-7033.

Jungmichel, S., Rosenthal, F., Altmeyer, M., Lukas, J., Hottiger, M.O. and Nielsen, M.L. (2013). Proteome-wide identification of poly(ADP-Ribosyl)ation targets in different genotoxic stress responses. Mol. Cell 52, 272-285.

Kapp, L.D., Abrams, E.W., Marlow, F.L., and Mullins, M.C. (2013). The integrator complex subunit 6 (Ints6) confines the dorsal organizer in vertebrate embryogenesis. PLoS Genet. 9, e1003822

Kim, Y.M., Watanabe, T., Allen, P.B., Kim, Y.M., Lee, S.J., Greengard, P., Nairn, A.C., and Kwon, Y.G. (2003). PNUTS, a protein phosphatase 1 (PP1) nuclear targeting subunit. Characterization of its PP1- and RNA-binding domains and regulation by phosphorylation. J. Biol. Chem. 278, 13819-13828

Kleiman, F.E., and Manley, J.L. (1999). Functional interaction of BRCA1-associated BARD1 with polyadenylation factor CstF-50. Science 285, 1576-1579.

Kleiman, F.E., and Manley, J.L. (2001). The BARD1-CstF-50 interaction links mRNA $3^{\prime}$ end formation to DNA damage and tumor suppression. Cell 104, 743-753.

Krishnakumar, R., and Kraus, W.L. (2010). The PARP side of the nucleus: molecular actions, physiological outcomes, and clinical targets. Mol. Cell 39, 8-24.

Lackford, B., Yao, C., Charles, G.M., Weng, L., Zheng, X., Choi, E.A., Xie, X., Wan, J., Xing, Y., Freudenberg, J.M., et al. (2014). Fip1 regulates mRNA alternative polyadenylation to promote stem cell self-renewal. EMBO J. 33, 878-889.

Li, W., Yeh, H.J., Shankarling, G.S., Ji, Z., Tian, B., and MacDonald, C.C. (2012). The tauCstF-64 polyadenylation protein controls genome expression in testis. PLoS One 7, e48373.

Lubas, M., Christensen, M.S., Kristiansen, M.S, Domanski, M. Falkenby, L.G., Lykke-Andersen, S., Andersen, J.S., Dziembowski, A., and Jensen, T.H. (2011). Interaction profiling identifies the human nuclear exosome targeting complex. Mol. Cell 43, 624-637.

Mandel, C.R., Bai, Y., and Tong, L. (2008). Protein factors in premRNA 3'-end processing. Cell. Mol. Life Sci. 65, 1099-1122.

Mayr, C., and Bartel, D.P. (2009). Widespread shortening of 3'UTRs 
by alternative cleavage and polyadenylation activates oncogenes in cancer cells. Cell 138,673-684.

Mbita, Z., Meyer, M., Skepu, A., Hosie, M., Rees, J., and Dlamini, Z. (2012). De-regulation of the RBBP6 isoform $3 / D W N N$ in human cancers. Mol. Cell. Biochem. 362, 249-262.

Meinhart, A., and Cramer, P. (2004). Recognition of RNA polymerase II carboxy-terminal domain by 3'-RNA-processing factors. Nature 430, 223-226.

Mueller, C.L., Porter, S.E., Hoffman, M.G., and Jaehning, J.A. (2004). The Paf1 complex has functions independent of actively transcribing RNA polymerase II. Mol. Cell 14, 447-456.

Nagaike, T., Logan, C., Hotta, I., Rozenblatt-Rosen, O., Meyerson, M., and Manley, J.L. (2011). Transcriptional activators enhance polyadenylation of mRNA precursors. Mol. Cell 41, 409-418.

Nazeer, F.I., Devany, E., Mohammed, S., Fonseca, D., Akukwe, B. Taveras, C., and Kleiman, F.E. (2011). p53 inhibits mRNA 3' processing through its interaction with the CstF/BARD1 complex. Oncogene 30, 3073-3083.

Ntini, E., Jarvelin, A.I., Bornholdt, J., Chen, Y., Boyd, M., Jorgensen, M. Andersson, R. Hoof, I., Schein, A., Andersen, P.R., et al. (2013). Polyadenylation site-induced decay of upstream transcripts enforces promoter directionality. Nat. Struct. Mol. Biol. 20, 923-928.

Ohnacker, M., Barabino, S.M., Preker, P.J., and Keller, W. (2000). The WD-repeat protein pfs2p bridges two essential factors within the yeast pre-mRNA 3'-end-processing complex. EMBO J. 19, 37-47.

Pashkova, N., Gakhar, L., Winistorfer, S.C., Yu, L., Ramaswamy, S. and Piper, R.C. (2010). WD40 repeat propellers define a ubiquitin-binding domain that regulates turnover of $\mathrm{F}$ box proteins. Mol. Cell 40, 433-443.

Penheiter, K.L., Washburn, T.M., Porter, S.E., Hoffman, M.G., and Jaehning, J.A. (2005). A posttranscriptional role for the yeast Paf1-RNA polymerase II complex is revealed by identification of primary targets. Mol. Cell 20, 213-223.

Proudfoot, N.J. (2011). Ending the message: poly(A) signals then and now. Genes Dev. 25, 1770-1782.

Reinhardt, H.C., and Yaffe, M.B. (2013). Phospho-Ser/Thr-binding domains: navigating the cell cycle and DNA damage response. Nat. Rev. Mol. Cell Biol. 14, 563-580.

Rozenblatt-Rosen, O., Nagaike, T., Francis, J.M., Kaneko, S., Glatt K.A., Hughes, C.M., LaFramboise, T., Manley, J.L., and Meyerson, M. (2009). The tumor suppressor Cdc73 functionally associates with CPSF and CstF $3^{\prime}$ mRNA processing factors. Proc. Natl. Acad. Sci. USA 106, 755-760.

Ryan, K., and Bauer, D.L. (2008). Finishing touches: posttranslational modification of protein factors involved in mammalian pre-mRNA $3^{\prime}$ end formation. Int. J. Biochem. Cell Biol. 40, 2384-2396.

Sakai, Y., Saijo, M., Coelho, K., Kishino, T., Niikawa, N., and Taya, Y. (1995). cDNA sequence and chromosomal localization of a novel human protein, RBQ-1 (RBBP6), that binds to the retinoblastoma gene product. Genomics 30, 98-101.

Sandberg, R., Neilson, J.R., Sarma, A., Sharp, P.A., and Burge, C.B. (2008). Proliferating cells express mRNAs with shortened $3^{\prime}$ untranslated regions and fewer microRNA target sites. Science 320, 1643-1647.

Shi, Y., Reddy, B., and Manley, J.L. (2006). PP1/PP2A phosphatases are required for the second step of Pre-mRNA splicing and target specific snRNP proteins. Mol. Cell 23, 819-829.

Shi, Y., Di Giammartino, D.C., Taylor, D., Sarkeshik, A., Rice, W.J., Yates, J.R., 3rd, Frank, J., and Manley, J.L. (2009). Molecular architecture of the human pre-mRNA $3^{\prime}$ processing complex. Mol. Cell 33, 365-376.

Simons, A., Melamed-Bessudo, C., Wolkowicz, R., Sperling, J., Sperling, R., Eisenbach, L., and Rotter, V. (1997). PACT: cloning and characterization of a cellular p53 binding protein that interacts with Rb. Oncogene 14, 145-155.
Stuparevic, I., Mosrin-Huaman, C., Hervouet-Coste, N., Remenaric, M., and Rahmouni, A.R. (2013). Cotranscriptional recruitment of RNA exosome cofactors Rrp47p and Mpp6p and two distinct TrfAir-Mtr4 polyadenylation (TRAMP) complexes assists the exonuclease Rrp6p in the targeting and degradation of an aberrant messenger ribonucleoprotein particle (mRNP) in yeast. J. Biol. Chem. 288, 31816-31829.

Takagaki, Y., and Manley, J.L. (2000). Complex protein interactions within the human polyadenylation machinery identify a novel component. Mol. Cell. Biol. 20, 1515-1525.

Takagaki, Y., Ryner, L.C., and Manley, J.L. (1988). Separation and characterization of a poly $(A)$ polymerase and a cleavage/specificity factor required for pre-mRNA polyadenylation. Cell $52,731-742$

Takata, H., Nishijima, H., Maeshima, K., and Shibahara, K. (2012). The integrator complex is required for integrity of Cajal bodies. J. Cell Sci. 125, 166-175.

Tian, B., and Manley, J.L. (2013). Alternative cleavage and polyadenylation: the long and short of it. Trends Biochem. Sci. 38, 312-320.

Topalian, S.L., Kaneko, S., Gonzales, M.I., Bond, G.L., Ward, Y., and Manley, J.L. (2001). Identification and functional characterization of neo-poly(A) polymerase, an RNA processing enzyme overexpressed in human tumors. Mol. Cell. Biol. 21, 5614-5623.

Vagner, S., Vagner, C., and Mattaj, I.W. (2000). The carboxyl terminus of vertebrate poly(A) polymerase interacts with U2AF 65 to couple 3'-end processing and splicing. Genes Dev. 14, 403-413.

Vethantham, V., Rao, N., and Manley, J.L. (2008). Sumoylation regulates multiple aspects of mammalian poly $(\mathrm{A})$ polymerase function Genes Dev. 22, 499-511.

Vo, L.T., Minet, M., Schmitter, J.M., Lacroute, F., and Wyers, F. (2001). Mpe1, a zinc knuckle protein, is an essential component of yeast cleavage and polyadenylation factor required for the cleavage and polyadenylation of mRNA. Mol. Cell. Biol. 21, 83468356.

Wallace, A.M., Dass, B., Ravnik, S.E., Tonk, V., Jenkins, N.A., Gilbert D.J., Copeland, N.G., and MacDonald, C.C. (1999). Two distinct forms of the $64,000 \mathrm{Mr}$ protein of the cleavage stimulation factor are expressed in mouse male germ cells. Proc. Natl. Acad. Sci. USA 96, 6763-6768.

Weitzer, S., and Martinez, J. (2007). The human RNA kinase hClp1 is active on $3^{\prime}$ transfer RNA exons and short interfering RNAs. Nature $447,222-226$

Xiang, K., Tong, L., and Manley, J.L. (2014). Delineating the structural blueprint of the pre-mRNA 3 '-end processing machinery. Mol. Cell. Biol. 34, 1894-1910.

$\mathrm{Xu}, \mathrm{C}$. , and Min, J. (2011). Structure and function of WD40 domain proteins. Protein Cell 2, 202-214.

Yang, Q., Nausch, L.W., Martin, G., Keller, W., and Doublie, S. (2014). Crystal structure of human poly(a) polymerase gamma reveals a conserved catalytic core for canonical poly(a) polymerases. J. Mol. Biol. 426, 43-50.

Yao, C., Biesinger, J., Wan, J., Weng, L., Xing, Y., Xie, X., and Shi, Y. (2012). Transcriptome-wide analyses of CstF64-RNA interactions in global regulation of mRNA alternative polyadenylation. Proc. Natl. Acad. Sci. USA 109, 18773-18778.

Yao, C., Choi, E.A., Weng, L., Xie, X., Wan, J., Xing, Y., Moresco, J.J., Tu, P.G., Yates, J.R., 3rd, and Shi, Y. (2013). Overlapping and distinct functions of CstF64 and CstF64tau in mammalian mRNA 3' processing. RNA 19, 1781-1790.

Zhang, F., Ma, T., and Yu, X. (2013). A core hSSB1-INTS complex participates in the DNA damage response. J. Cell Sci. 126, 48504855.

Zhao, W., and Manley, J.L. (1996). Complex alternative RNA processing generates an unexpected diversity of poly(A) polymerase isoforms. Mol. Cell. Biol. 16, 2378-2386. 